\title{
Aproximación a la compresión del gerenciamiento de proyectos públicos
}

\author{
Compression approach of the management of public projects \\ Recibido: 23-04-2019 • Aprobado: 28-07-2020 • Página inicial: 47 - Página final: 68 \\ Carlos Alberto Restrepo Carvajal*
}

\begin{abstract}
Resumen: el artículo revisa la postura de diferentes autores que centran su atención en la gestión de proyectos públicos y en la carencia de políticas públicas orientadas a estos, la cual debe estar en función de la misión, visión y objetivos estratégicos. Por lo tanto, se debe asegurar que los resultados esperados (objetivos del proyecto) estén alineados con ellos, garantizando el aporte de valor (bienestar). No obstante, para hacer que esto suceda, se debe realizar una revisión interna que refleje las brechas existentes en materia de gestión de proyectos, la cual se desarrolla mediante la evaluación del nivel de madurez en gerenciamiento de proyectos (portafolio, programas y proyectos).
\end{abstract}

Palabras clave: gerencia de proyectos; proyectos públicos; indicadores; políticas públicas.

\begin{abstract}
This article reviews the position the different authors that focus on public management projects, and in the lack of public policies aimed to this management, which must be in function of the mission, vision and in the strategic objectives, therefore, it is possible to guarantee the expected results (project objectives) are aligned to this, ensuring the create of value (well-being). However, in order to fulfil this, it is necessary to conduct an internal review that reflects the existing gaps in project management, which is developed by assessing the level of maturity in project management (portfolio, programs and projects).
\end{abstract}

Keywords: Project management; public projects; indicators; public policy.

JEL: O29 - Z19

\footnotetext{
* PMP, Gerente Desarrollo 10XPRO, candidato a Doctor en Dirección de Proyectos, Centro Panamericano de Estudios Superiores, México. Magíster en Ingeniería, Magíster en Administración y Especialista en Gerencia de Proyectos. carlosrestrepo@une.net.co - carlos.restrepo.carvajal@gmail.com ORCID: https://orcid.org/0000-0002-1889-3340
} 


\section{Contexto Pestroos.c.}

\section{Abordagem de compressão da gestão de projetos públicos}

Resumo: o artigo revê a posição de diversos autores que focam sua atenção na gestão de projetos públicos e na carência de políticas públicas orientadas para estes, que devem estar pautadas na missão, visão e objetivos estratégicos. Portanto, deve-se garantir que os resultados esperados (objetivos do projeto) estejam alinhados com os mesmos, garantindo a contribuição de valor (bem-estar). Porém, para que isso aconteça, deve ser realizada uma revisão interna que reflita as lacunas existentes na gestão de projetos, que se desenvolve avaliando o nível de maturidade em gestão de projetos (portfólio, programas e projetos).

Palavras-chave: gestão de projetos; projetos públicos; indicadores de gestão e políticas públicas. 


\section{Introducción}

Un desafío constante para cualquier país es la definición y puesta en marcha de unas prácticas adecuadas de Gerenciamiento de Proyectos, que favorezcan y potencien la gestión efectiva y el éxito de estos en función de sus objetivos estratégicos. Es así, como cada vez es más fehaciente y necesaria la definición de una política pública que estandarice la gestión de proyectos, considerando el ciclo de vida y gerenciamiento per se de estos.

Es primordial para avanzar en la gestión efectiva de proyectos públicos que los altos dirigentes establezcan unos criterios rigurosos, diáfanos, pertinentes, creíbles y socializados para las etapas de inicio y cierre de proyectos. Es frecuente observar que este tipo de proyectos no posee un cierre gerencial, sino que estos son literalmente abandonados.

En el gerenciamiento de proyectos públicos se soslaya la necesidad de establecer cuáles son los factores de éxito del proyecto, asociados a los requerimientos de los interesados. Así mismo, se adolece de una comprensión clara del caso de negocio y de la estrategia organizacional. Por lo tanto, no se está evaluando el impacto real de los proyectos desde un análisis sistémico.

\section{Gestión de proyectos públicos}

Los gobiernos tradicionales se encuentran en serias dificultades para reaccionar con rapidez y pertinencia ante un entorno complejo y dinámico que involucra a una ciudadanía cada vez más reflexiva y crítica, la cual opera bajo el concepto de valor compartido y no se conforma con la provisión de servicios públicos (Blanco y Gomá, 2002). Es imperioso que los proyectos públicos estén direccionados al logro de los siguientes planes:

- Planes estratégicos: mecanismos para la planificación de estrategias de desarrollo socioeconómico a mediano y largo plazo en un territorio determinado.

- Presupuestos participativos: herramienta para la participación de la ciudadanía en la elaboración de los presupuestos municipales.

- Agendas locales: programa de acciones para conseguir que un territorio determinado tenga un futuro sostenible.

- Planificación participativa: espacio dado para la participación de la comunidad en diversos temas de desarrollo. 


\section{Contexto Rastopo, c.}

En Colombia, las decisiones de inversión en el sector público no siempre están ligadas a estrategias estatales, sino a políticas, las cuales deberían de ir ligadas a planes de acción, planes de desarrollo, planes de ordenamiento, agrupándose en portafolios y programas, que, a su vez, conformarían el Plan Nacional de Desarrollo, en el cual deben estar las iniciativas que aporten valor a los objetivos estratégicos de la región y/o país. Para Gómez et al. (2012), los planes generales o planes de acción se entienden como un esquema general de trabajo que define, a grandes rasgos, las prioridades, los lineamientos básicos de una gestión y el alcance de sus funciones; estos se traducen en instrumentos que hacen realidad la materialización de los Planes de Desarrollo, los cuales se convierten en ideas que van a orientar y condicionar los portafolios, programas y proyectos.

De acuerdo a Pilkaité \& Chmieliauskas (2015), los planes de acción, los programas y los proyectos se deben imbricar para integrar un Plan de Desarrollo, sea del orden nacional o regional, el cual debe seguir los principios de financiación del Estado. Desafortunadamente, las prácticas de gestión pública de proyectos no son las más adecuadas, presentando tres causas importantes: la primera se asocia con la débil coordinación e integración entre los proyectos, sean de orden regional o nacional; la segunda, con la ausencia de directrices de gobierno apropiadas en temas de gestión de proyectos; y la tercera, con personal inadecuado para gerenciar proyectos.

Evans (2009) argumenta que transformar el Estado industrial en uno de competencia, partiendo del hecho de que con mayor frecuencia el gobierno define políticas de asociación empresarial para su mantenimiento, promoción y expansión a una economía global para traer beneficios, donde este cambio hacia la modernización se encuentra soportado en: liderazgo, cambio del estado naturaleza política, identificados como agentes empresariales de la globalización, menor diferencias ideológicas entre los partidos políticos y la internacionalización de la política vigente. Es así como la modernización de las políticas públicas implica competitividad a través de la capacitación y conservación de su equipo de trabajo alrededor de sus funciones, sin que con esto se limite su creatividad, dejando de lado la intención del favorecimiento especial de algunos interesados. En este sentido, los proyectos públicos resultan ser muy interesantes, debido a que es la unión entre la estrategia de Gobierno y el modelo de Desarrollo Nacional. Análogamente, con el sector privado sería el empalme entre la estrategia y el modelo de negocio. 
El proceso de modernización de las políticas públicas ha transformado las funciones y roles del gobierno, hizo que bienes y servicios públicos los administraran, en conjunto, entidades estatales con semi-autonomía y privadas prestadoras de servicios públicos con estrategias regionales a nivel local, naciendo una amplia red de gobierno a nivel mundial, cuyos desafíos radican en la gestión de los proyectos públicos para adaptarse a la nuevas políticas públicas de satisfacer las necesidades de la red de gobierno (Pierre \& Peters, 2000). En el contexto colombiano, no se ha logrado armonizar de manera integral y a largo plazo las políticas públicas con los roles del gobierno, ni las necesidades de competitividad; por el contrario, han primado los beneficios particulares de personas y partidos sobre el bienestar de la región y/o del país.

La gestión administrativa de proyectos públicos ha estado presente desde siempre; es así, como la construcción de edificaciones importantes -por ejemplo, las obras magnánimas de los grandes imperios- son el reflejo de este hecho. Los resultados aún resultan impresionantes, pero en general requirieron una gran gestión sobre las diferentes restricciones, a saber: alcance, tiempo, costo, calidad, etc.

Con el trascurrir de los años, esta gestión fue "puliéndose" y fue necesario un cambio para afrontar las nuevas realidades del mundo, sus necesidades y exigencias. Aunque la introducción de cambios ha sido tan paulatina y escalonada, como diversa y flexible, se pueden identificar tres grandes ejes, sobre los que recae las principales modificaciones: el quién (el gestor o gerente), el cómo (metodología) y el para qué (necesidad).

El gestor (de burócrata a gerente): El primer debate que se presenta para la ejecución de proyectos que beneficien a una sociedad, se concentra sobre la selección del mejor gestor: la función pública o la iniciativa privada. Esta disyuntiva está presente durante toda la evolución de la gestión pública. En un primer momento (siglo XIX), y frente a la desigualdad en la distribución de los capitales, esta labor fue asumida enteramente por el Estado (un Estado empresario), quien con una activa participación intentaba resarcir "los errores del mercado" (García Sánchez, 2007).

Posteriormente (década de los 70 del siglo XX), se generan alertas sobre la ineficacia de esta labor, asociadas a la burocracia y a la corrupción; y, una vez más, se critican los resultados evidenciando "los fallos del Estado" (García Sánchez, 2007). Años más tarde, empieza a aparecer una serie de guías para el gerenciamiento de proyectos, con el fin de dar los primeros pasos hacia una estandarización de su práctica, favoreciendo su logro y gestión. 


\section{Contexto Rastopo, c.}

Una de las mayores innovaciones en la gestión de proyectos de los últimos años es la humanización del Gestor de Proyectos. Se pasa de un Gestor de Proyectos con perfil técnico e implementador, conocedor de la materia, inflexible en las decisiones, enfocado únicamente al resultado, no líder visible en el equipo y en la organización, a un Gestor de Proyectos disciplinado, comprometido, con conocimiento, líder visible, flexible, tolerante con habilidades de comunicación efectiva y de coach del equipo, el hecho más importante es que se centra en el recurso más importante del proyecto y la organización: las personas.

El cómo: se han dado cambios importantes en la manera de abordar el gerenciamiento de proyectos; es así como actualmente existen diferentes escuelas de pensamiento, de las cuales resaltan el Project Management Institute (PMI), International Project Management Association (IPMA) y Project in Controlled Enviroments (PRINCE). Es de anotar, que estas escuelas han establecido una serie de guías para el gerenciamiento, donde el gerente de proyectos, de acuerdo con sus habilidades, define cuáles serán implementadas. De igual manera, es evidente la necesidad del establecimiento de una política pública como medio de control al gerenciamiento de los proyectos. Un objetivo importante de la política pública en materia de gestión de proyectos debería ir más allá de acciones puntuales de control, debería garantizar o guiar la gobernabilidad en la adopción de estrategias como la gestión de portafolios, programas y proyectos.

Las políticas públicas son instrumentos que le permiten al Estado el control social. A través de directrices estatales, los Estados y entes territoriales pueden ejercer autoridad frente a un colectivo, como una manera de abordar un tema específico y de interés público. Es de anotar, que más que un proceso técnico metódico, las políticas públicas son el producto de múltiples acciones conjuntas que deben ser coordinadas eficientemente por el Estado para garantizar la vigilancia de los recursos (Gómez, 2012).

Desde un enfoque más técnico, las políticas públicas son sistemas complejos de análisis, decisión y control que deben sortear la racionalidad de las decisiones de la gestión de los gobiernos, con el propósito de reducir la incertidumbre y favorecer la gestión de los riesgos, asegurando en todo momento los fundamentos del orden social. En este orden de ideas, una política pública debe estar enmarcada en un amplio proceso de participación social que favorezca la discusión política formal (Lahera, 2004). Adicionalmente, debe dar lugar a una fundamentación amplia de contenidos, tener claridad en los objetivos, elegir la mejor opción de financiamiento, realizar una evaluación costos versus beneficio social y garantizar la funcionalidad de los instrumentos de intervención. 
Según Crawford \& Helm (2009), la gobernabilidad es un aspecto complejo y concerniente al sector público con una necesidad política vivida para suplir las necesidades de las partes interesadas, mediante la rendición de cuentas, informes de control, la transparencia y la aplicación efectiva de los instrumentos de control, direccionados al logro de las estrategias públicas. En gran medida, las iniciativas relativas al gobierno son generadas por una necesidad, que no siempre es clara, de mejora de una parte de la sociedad. Necesidad que generalmente se refleja en proyectos y programas. La gestión de proyectos, por ejemplo, de infraestructura está determinada por enfoques que la subrayan como un proceso racional, bajo protocolos abiertos y planificados. Sin embargo, según Verweij (2015) existe poca certeza de una comprensión realista de la gestión de proyectos, debido a que su planeación y ejecución se presenta de manera aislada con su contexto físico y social.

Más recientemente, se propone un híbrido entre los dos sectores, apareciendo las Asociaciones o Alianzas Público Privadas (APP) que se refieren a contratos entre el gobierno y un ente privado con el propósito de culminar un proyecto que tradicionalmente ha sido atendido por el Estado, en donde este último se apoya en recursos privados. Esta alternativa permite al socio público introducir con mayor rapidez y flexibilidad tecnología e innovación, tener mayor control sobre sus presupuestos, diversificar sus inversiones y complementar sus limitaciones. Al mismo tiempo, permite al aliado privado desarrollar sus capacidades, aumentar su participación en el mercado y compartir los riesgos del proyecto según el modelo elegido (Alborta, Stevenson y Triana, 2011). Aunque la participación del sector privado en proyectos públicos no es nueva, el modelo APP amplía y flexibiliza esta relación, proporcionando un marco participativo para la provisión de infraestructura y servicios públicos.

En este sentido, la gestión de proyectos en un contexto regional tiene como fin dar respuesta a dos escenarios simultáneos: las exigencias globales, que buscan estandarización en pro de integrar económica, social, política y culturalmente a todos los países; y las necesidades locales, que requieren modelos ajustados a comunidades específicas y localidades puntuales (Jordán y Simioni, 2003). En dicha gestión, es evidente la necesidad de que la gerencia de proyectos públicos incorpore modelos dinámicos de análisis de entorno, toda vez que estos son permeables a las condiciones y variables exógenas.

En la actualidad, el principal reto de la Gestión de Proyectos es la globalización, que obliga a que esta se expanda a entornos internacionales y multiculturales; otro reto, es lograr resultados más rápidos y de alta calidad en un ambiente de alta incertidumbre que multiplica los riesgos. 


\section{Contexto Rastopo, c.}

Por otra parte, es importante considerar, en el ámbito de lo público, la evolución que se ha venido presentando en los últimos años: pasar de un Estado empresario (con activos productivos) a un Estado regulador (básicamente normativo, sin activos). Esta es la base del actual capitalismo, que considera la total privatización de los activos públicos. Por ejemplo, Colombia tiene un portafolio de empresas del Estado que está siendo revisado por influencia del FMI (Fondo Monetario Internacional), BID (Banco Interamericano de Desarrollo), CEPAL (La Comisión Económica para América Latina) y, sobre todo, de la OCDE (Organización para la Cooperación y el Desarrollo Económicos); este último tiene exigencias en este sentido para poder aceptar la solicitud de ingreso.

El para qué: en los últimos años se han dado una serie de cambios profundos y acelerados en las regiones, obligando a los gobiernos a incorporar en sus indicadores asuntos que antes se consideraban tangenciales, con el fin de suplir las demandas y necesidades de desarrollo: la diversidad cultural, la cooperación o la sostenibilidad. De igual manera, como lo menciona Blanco y Gomá (2002), es menester que los proyectos estén alineados a los planes estratégicos y al desarrollo de un país.

Considerando lo expuesto anteriormente, se comprende que ya no es posible abordar, caracterizar y enfrentar situaciones o problemas colectivos con modelos impostados; y, por el contrario, se entiende el bienestar comunitario como una consecuencia del desarrollo local bajo una mirada holística, multidisciplinaria, participativa e integral de dicha comunidad (Figueroa, 2005). En este orden de ideas, la Gestión de Proyectos Públicos debe incluir modelos que permitan la definición de las necesidades reales y contextualizadas de los interesados, sean estos: comunidades, empresas, gobiernos, etc.; y, así mismo, metodologías para la estimación y valoración de los impactos del proyecto, sean positivos y/o negativos.

Verweij (2015) afirma que la gerencia de proyectos está definida por un enfoque adiabático, es decir, por un sistema cerrado, lo cual no toma lugar en la realidad. Los proyectos interactúan con un sinnúmero de dimensiones, entre las cuales se encuentran el contexto social y psicológico de las personas; por ende, este gerenciamiento debe planearse desde el análisis de sistemas abiertos.

Este mismo autor aborda el tema de las APP, definidas como mecanismos para mejorar el desempeño de los proyectos de infraestructura pública. En su investigación encontró que este tipo de alianzas no han procurado un mejor gerenciamiento de los proyectos; de hecho, es evidente las debilidades en 
planeación, concentrándose principalmente en la fase de ejecución y reflejando ineficiencias en el uso de los recursos y en la administración de los riesgos.

La incertidumbre de este tipo de alianzas puede conducir a la reducción del intercambio de información, negociaciones ineficientes y baja disposición a invertir, factores que impactan negativamente al desarrollo de los proyectos. En este sentido, Canoy, Janssen, Vollaard, van Bork y Ham (2001) exponen que la solución más apropiada para esta condición normal de inseguridad, es anticiparse a los riesgos potenciales a través de su identificación oportuna y, adicionalmente, permitir que la cooperación establezca las condiciones que permitan alcanzar beneficios compartidos. Además, sugiere la construcción de Sistemas de Autorización de Trabajo (SAT) para la definición de roles y responsabilidades.

De las APP se pueden obtener beneficios, favoreciendo el aprovechamiento de oportunidades, a saber: mejoría en los procesos de planificación asociados al intercambio de información -lecciones aprendidas-, incrementos en la eficiencia de los componentes relacionados en la Estructura de Desglose de Trabajo (EDT), intercambios de experiencia que favorecen los logros del proyecto, mejor apalancamiento de la estructura de costos, debido a que las partes podrán concentrarse en lo que mejor saben hacer; por lo tanto, se potencian las economías de escala y alcance y, finalmente, se desarrolla una mejor gestión de los riesgos.

Akhmetshina \& Mustafin (2015) expresan que actualmente varios países han implementado las APP como estrategia de desarrollo social en sus políticas públicas; por ejemplo, en Alemania la proporción de las organizaciones que reciben fondos presupuestales para estos fines es del 8,8\%, y en Bélgica es del 12,7\%. Manifiestan que en Finlandia el Estado promueve la innovación a través de la "Fundación de Apoyo a la Invención", donde se establecen este tipo de alianzas para participar activamente en la configuración del sistema de innovación, es decir, proporcionan asesoramiento empresarial y asistencia financiera en el desarrollo de la gestión de la innovación, la evaluación de nuevos productos, patentes y comercialización de patentes. Por otra parte, Klijn (2009) asocia las APP como instrumentos de capitalización del Estado y no como un sistema de Gerenciamiento de Proyectos públicos.

Wu, Huang, Luo \& Li (2014) hacen énfasis en que los proyectos públicos desempeñan una función muy importante en la economía de la República Popular China, y en este sentido, en el año 2004, el Gobierno -mediante el 


\section{Contexto Rastopo, c.}

Consejo de Estado- decidió favorecer la implementación de un modelo de Agentes de Sistemas de Construcción (ACS) para los proyectos públicos sin ánimo de lucro. En esta integración de sistemas, el eje fundamental es la incorporación y comprensión de los ciclos de vida, principalmente el ciclo de vida extendido del proyecto.

Por su parte Shiferaw \& Klakegg (2012) describen, en su estudio desarrollado para la Oficina Gubernamental de Comercio del Reino Unido (OGC), que la carencia de una clara vinculación entre el proyecto y las prioridades estratégicas de la organización es una de las principales causas de proyectos fracasados. No obstante, el número de proyectos que no puede satisfacer las necesidades y prioridades del público también está aumentando.

Considerando el análisis comparativo de investigaciones realizadas en Europa, abordado desde perspectivas diferentes en la aplicación de instrumentos en proyectos de salubridad del sector público, existen instrumentos convencionales que están ligados a definir la autoridad, capacidad, incentivos por productividad, contemplando métricas operativas como la gestión del tiempo, costo y alcance de las actividades que se van a desarrollar durante todo el proyecto, lo cual conduce a considerar sólo estas tres restricciones, situación que conlleva a que la gestión de los proyectos no sea exitosa (Kapsali, 2011).

Como efectos de una inadecuada gestión de proyectos, a nivel mundial se puede mencionar proyectos sin éxito como el de desarrollo más grande de África al final del 2003: el oleoducto Chad-Camerún, el cual costó USD \$4,200 millones. A pesar del acuerdo con el gobierno de Chad, que, para ser financiado por el Banco Mundial, los ingresos sustanciales del petróleo se deberían redirigir a la reducción de la pobreza, el gobierno se negó a asignar recursos suficientes para la reducción de la pobreza crítica, en educación, salud, infraestructura, desarrollo rural y la gobernanza. En el año 2005, el gobierno de Chad anunció que el dinero del petróleo iría hacia el presupuesto general y el rearme de las fuerzas armadas, en contra de los objetivos originales del proyecto. Este empréstito, de acuerdo con el gobierno de Chad, fue aclamado como "innovador" debido a que canalizaba ingresos para los pobres, sin que estos finalmente pudieran beneficiarse de acuerdo con el objetivo original (Asmamaw \& Ole, 2012).

En Yamoussoukro, Costa de Marfil, Félix Houphouët Boigny construyó la iglesia más grande del mundo (Basílica de la Virgen de Paz de Yamoussoukro) con un costo de US $\$ 360$ millones; en el Libro Guinness Récords figura el proyecto como la iglesia más grande del mundo. Sin embargo, actualmente, 
permanece vacía. Otro ejemplo de proyectos, se relaciona con el gobierno de Uganda, con la ayuda del Banco Mundial y el Banco Africano de Desarrollo, construyó una represa de 200-megavatios cerca de las cataratas de Bujagali en el Nilo. La represa tenía un efecto devastador en las comunidades de la zona. La contracorriente sumergió una enorme área de tierra cultivable y establecida, forzando a la migración y el reasentamiento de gran cantidad de personas (Asmamaw \& Ole, 2012).

Wu et al. (2014) concluyen que el modelo ACS impactó positivamente con la intención de subsanar un sinnúmero de dificultades en el sector constructor, favoreciendo mejores resultados de gestión, potenciando el desarrollo de la economía local. De igual manera, estos autores destacan el SHE (Safety, Health and Environment Principle). La seguridad social, ambiental y ocupacional está ligada al éxito del proyecto, lo cual está inmerso en las buenas prácticas de sostenibilidad (Swan \& Khalfan, 2007). En corolario con lo anterior, la gestión de proyectos debe incluir unos lineamientos muy diáfanos en el tema de Responsabilidad Social Corporativa.

Restrepo y Alzate (2013) presentan en la Figura 1 un diagrama de relaciones de todos los interesados de un proyecto. Este análisis conlleva a un entendimiento de las necesidades de estos interesados, que en última instancia soportan la construcción del enunciado del alcance del proyecto y del producto. Es de anotar, que en la gestión pública resulta más interesante y complejo este análisis, debido a la interacción del sistema abierto con un sinnúmero de variables del entorno.

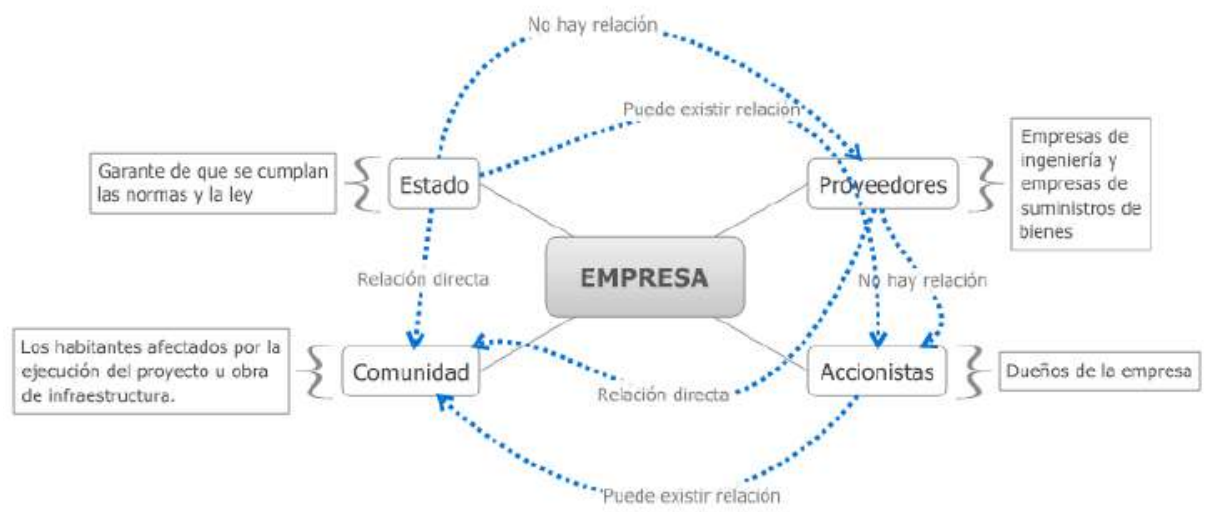

Figura 1. Diagrama de relaciones RSC a proyectos.

Restrepo y Alzate (2013). 


\section{Contexto Rastopo, c.}

La Responsabilidad Social Corporativa cubre tres dimensiones: desempeño económico, administración ambiental e inversión social. En este aspecto, el teórico Jhon Elkington (como se citó en Wilenius, 2005) sugirió un modelo triangular, Figura 2, el cual ha sido usado en diferentes corporaciones privadas y estatales como herramienta para balancear las metas netamente económicas con una visión social y ambiental.

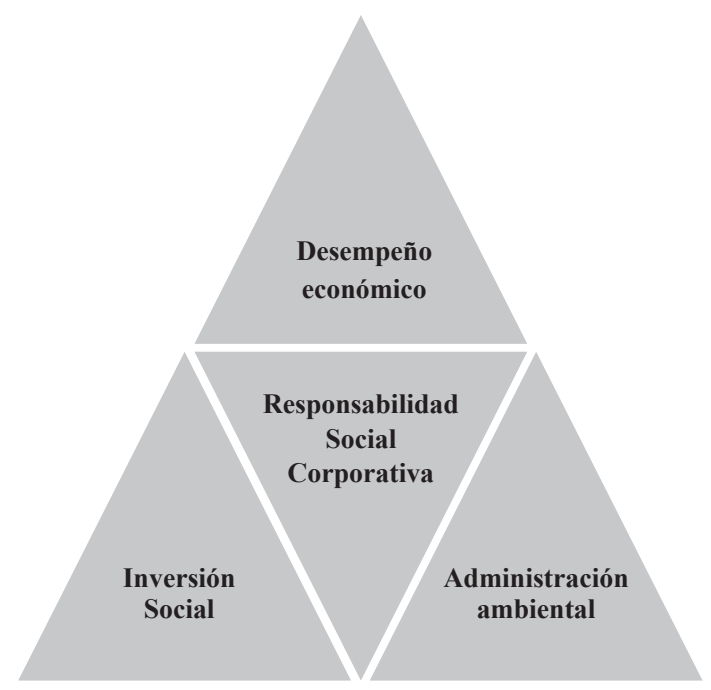

Figura 2. Modelo Triangular de Responsabilidad Corporativa.

Adaptado de Wilenius (2005).

La gestión pública de proyectos gerencia las relaciones entre los diferentes interesados en un proyecto, lo cual implica negociación, concertación de acuerdos y establecimiento de contratos entre las partes; por lo tanto, el desarrollo de dichas redes por medio de la asistencia tecnológica o el fortalecimiento institucional, y su correspondiente flujo de información, hace parte de la gestión de la gerencia del proyecto, donde se garantice la gestión del conocimiento (Schweinheim, 1998). Por su parte, Crawford \& Helm (2009) aseveran que el sector público está caracterizado por una complejidad intrínseca del resultado de la interacción de los grupos de valor, lo cual se refleja en problemas de incertidumbre y, por tanto, en los riegos. De igual manera, los proyectos públicos están sujetos a presiones políticas, escrutinio público y rendición de cuentas.

Mihaescu \& Tapardel (2013) investigaron el contexto del gerenciamiento en Rumania e identificaron las siguientes características: 
- La existencia de un marco jurídico normativo que determina las actividades en general. Este está diseñado para las instituciones públicas que llevan a cabo proyectos.

- El uso de los recursos públicos con el fin de llevar a cabo proyectos gubernamentales financiados por el presupuesto estatal, lo cual aumenta la responsabilidad de los políticos y gestores públicos.

- La existencia de un gran número de actores involucrados (instituciones gubernamentales, agencias, administradores públicos, grupos de interés y representantes políticos) y la rendición de cuentas a la sociedad.

- La dificultad de establecer prioridades y diferenciar los proyectos gubernamentales.

- Capacidad de la organización para implementar proyectos.

En su investigación, Pulmanis (2013) define que los gobiernos de algunas jurisdicciones brindan orientación sobre la forma de evaluar las propuestas, el uso de costos y beneficios, antes de comprometer fondos significativos de su presupuesto. Por ejemplo, los gobiernos de Australia, Nueva Zelanda, Reino Unido y Estados Unidos proporcionan guías sobre temas y técnicas que deben tenerse en cuenta al momento de evaluar nuevas políticas de regulación, programas y proyectos. Finalmente, Pulmanis (2013) expone las siguientes prácticas de gerenciamiento para proyectos públicos:

- Incrementar la capacidad y las habilidades profesionales para los equipos de Gerenciamiento de Proyectos.

- Definir apropiadamente la estructura organizacional para la elaboración del proyecto y la implementación del mismo.

- Las herramientas de Gerenciamiento de Proyectos y las técnicas deberían ser aplicadas gradualmente y obligatorias para proyectos públicos de gran escala.

La investigación dirigida por Pilkaité \& Chmieliauskas (2015) se centró en identificar los principales problemas que surgen en los proyectos públicos de Lituania, resultando que los más importantes están asociados a debilidades en la planificación, en la identificación de las restricciones y en el análisis del entorno. De sus resultados, se resaltan las funciones básicas de una Oficina de Gerenciamiento de Proyectos, Project Management Office (PMO), enunciadas a continuación (Project Management Institute (PMI), 2017):

- Manejar los recursos organizacionales.

- Desarrollar la metodología y práctica de la gestión del proyecto.

- Clases particulares, tutorías, entrenamiento en la gestión del proyecto. 


\section{Contexto Bestroo, c.}

- Monitorear el cumplimiento de las normas, estándares, procedimientos y reportar el estado del proyecto.

- Coordinar la comunicación y asegurar la calidad.

- Crear procesos de patrimonio organizacional, políticas del proyecto, plantillas y procedimientos.

- Funciones adicionales dependiendo del PMO: facturación del servicio, participación de analistas de negocios.

En consonancia con las funciones de la PMO, Istrate, Marian \& Ferencz (2014) definen tres direcciones de investigación para alcanzar el éxito de un proyecto, a saber:

- ¿Cómo? Lo que se justifica a través de funciones de grado inferior y hace hincapié en la construcción del árbol de decisiones.

- ¿Para qué? Justifica la contribución de las funciones de un grado inferior para los principales en una función compleja de la función de un grado inferior.

- ¿Cuándo? El tiempo para alcanzar la disyunción. Los momentos en los que la mayoría de las funciones se deben satisfacer simultáneamente.

Aunado a lo anterior, Cavalcante, Sousa, Milito y Oliveira (2014) afirman que la oficina de gestión de proyectos, PMO, es entendida por los administradores públicos como un elemento primordial para la alineación de los proyectos con los objetivos organizacionales. Además, como una herramienta para el seguimiento sistemático de los proyectos en desarrollo y para la creación de criterios de selección de proyectos.

En la investigación realizada por Fitsilis \& Chalatsis (2014), en Grecia, sobre organizaciones públicas, para revisar las prácticas de Gerenciamiento de Proyectos encaminadas a una gestión exitosa, mediante el uso de encuestas semiestructuradas, se encontró que existen dos grandes grupos que inhiben la implementación de sistemas de Gerenciamiento de Proyectos. El primero de ellos está relacionado con los ajustes de los estándares de la estructura de Gerenciamiento de Proyectos; el otro, se asocia con los factores que inhiben el ambiente interno de estos grupos de trabajo. En este orden de ideas, Florescu (2012) establece que el éxito en el Gerenciamiento de Proyectos públicos depende de la medición del desempeño del gerenciamiento de los procesos al interior de organismos públicos. Así mismo, en estos no resulta palmaria la construcción de baterías (grupos) de indicadores que permitan un seguimiento y control objetivo y metódico a la calidad de los procesos, anidados a objetivos estratégicos y de proyectos. 
El Gerenciamiento de Proyectos debe estar en un contexto tal, que sea posible definir herramientas e instrumentos de gerenciamiento que favorezcan y potencien el éxito de proyectos y la gestión efectiva de los mismos. En este análisis, Florescu (2012) afirma que es menester que el sector público defina un modelo de gestión de proyectos que incorpore la complejidad de estos y los riesgos asociados, considerando la estructura organizacional y funcional. En este mismo sentido, Cavalcante et al. (2014) afirman que los entes públicos deben enfocarse en el seguimiento y control de las restricciones y objetivos del proyecto. En este orden de ideas, los proyectos son de carácter temporal, único y multifuncional, permeados por los procesos de innovación, desarrollo de nuevos servicios y productos, y la mejora o adaptación al entorno de la organización.

En la investigación desarrollada por Roratto y Dotto (2012) se presenta el resultado del diagnóstico de los principales factores que podrían afectar negativamente el proceso de Gerenciamiento de Proyectos en una organización pública en Brasil, considerando los siguientes ejes la base para la construcción de una política pública de gestión de riesgos: la falta de infraestructura de la organización, desprestigio moral del Estado, desequilibrio entre la función pública, limitación legal excesiva, la continuidad administrativa, poca flexibilidad presupuestaria, el clima y la cultura organizacional constantemente en conflicto, la falta de profesionalismo en el nombramiento de personal y los retrasos en el proceso de adquisición. De igual forma, estas dificultades hacen que el sector público generalmente se muestre resistente a la adopción de buenas prácticas de Gerenciamiento de Proyectos.

En el estudio realizado por Kuprenas, Smith \& Madjidi (1999), en donde se referencia a La Mesa de Ingeniería de la ciudad de los Ángeles (EEUU), la cual es la encargada de planear, diseñar, construir y direccionar los proyectos de esta ciudad, plantea que La Mesa decidió elaborar e implementar un plan de mejora en el gerenciamiento de proyectos, debido a debilidades que se encontraron en la definición de la estructura organizacional y en la asignación de roles y responsabilidades de los miembros de equipo. Esta investigación recomienda la implementación de una estructura de pensamiento ágil en la gestión de proyectos públicos, soportada en una revisión pormenorizada de los procesos, con el fin de eliminar desperdicios y mejorar el desempeño. Para este fin se establece lo siguiente:

- Elaboración de formatos - para establecer acuerdos - asociados a las restricciones del proyecto, como tiempo y costos, para aquellos procesos que se consideren pertinentes. 


\section{Contexto Rastopo, c.}

- Definición de acuerdos diáfanos entre La Mesa de ingenieros y el cliente, en los cuales se define la gestión del alcance y las responsabilidades de ambas partes.

- Establecimiento de medidas de desempeño mediante el diseño e implementación de cuadros de control (Gestión de Indicadores estratégicos).

- Construcción de instrumentos que permitan la medición y el reporte del desempeño del proyecto (Gestión del Valor Ganado).

En el Gerenciamiento de Proyectos públicos es palmaria la necesidad de incorporar una gestión de riesgos más asertiva y dinámica, que incluya todo el ciclo de vida del proyecto, y el análisis de procesos, denominado Supplier, Inputs, Process, Outputs and Customers (SIPOC) sean pertinentes. Respecto a este tema, Yang (2007) define un riesgo como un juicio incorrecto de algo impredecible; además, los riesgos en proyectos públicos deben ser gerenciados y controlados.

Reyes y Gonzalez (2014) complementan el tema de la gestión de los proyectos, indicando que la estructura matricial es, tal vez, la más usada por las organizaciones que desarrollan proyectos, debido a que se logran economías de escala, al compartir y optimizar los recursos. Esta estructura permite reportar a un líder de proyecto y a un gerente funcional, pero así mismo es causa de conflictos, posibles esfuerzos duplicados y menor lealtad entre los miembros del equipo. Una situación que se observa con frecuencia en las reuniones de seguimiento de proyectos, especialmente en el sector público, concierne a que estas se concentran en los análisis de ejecución/planificación, es decir, se enfocan en el pasado, obviando que la gestión de los riesgos es la mejor y la única manera de prever y corregir desviaciones del proyecto.

Asmamaw \& Ole (2012) sugieren que los Estados establezcan un vínculo transparente entre las políticas y planes de desarrollo con la gestión de proyectos, mediante un sistema obligatorio de gestión de proyectos que permita el correcto proceso de selección y priorización de proyectos de inversión pública, con el fin de favorecer el éxito de los mismos. En este punto, se analiza la necesidad imperiosa de enlace entre los proyectos, las políticas, las estrategias, las necesidades y las prioridades públicas; de tal forma, que se logre un adecuado proceso de toma de decisiones por parte de los actores gubernamentales.

Por su parte, Schweinheim (1998) establece que es imperativo que las organizaciones, sin considerar si son públicas o privadas, definan unas competencias directivas soportadas en las siguientes dimensiones: funciones 
gerenciales de equipo, habilidades gerenciales, el Know How en tecnologías de gestión, construcción de un marco de referencia de la inversión en capital humano y en la formación de capital social. Este mismo autor enuncia uno de los puntos en común entre diversos proyectos exitosos del sector público, fundamentado en la planeación estratégica, debido a que una vez constituido el análisis de la situación y de tener establecido la misión y visión, es posible modificar las estrategias y objetivos asociados a los cambios del entorno y del sector.

El análisis estratégico contribuye a la función gerencial de la gestión de la agenda institucional, la cual es la base para el diseño e implementación del proceso de definición de unas políticas públicas para la gestión de proyectos, para este fin, la gerencia pública se debe encargar de identificar los problemas a resolver, a través de la elaboración de un diagnóstico que mida la gestión sobre el ciclo de vida de gerenciamiento y el ciclo de vida del proyecto. Se sugiere que este diagnóstico lo desarrolle personal externo a la organización, para favorecer los procesos de transparencia y ética.

\section{Conclusiones}

El factor común en los artículos revisados resalta el hecho de que aún los Gobiernos continúan soslayando la necesidad de una política diáfana, rigurosa y metódica para el gerenciamiento de proyectos, donde se defina una clara distinción entre proyectos exitosos y gestión exitosa, aclarando, que se deben buscar ambos aspectos. Los proyectos exitosos son aquellos donde es palmaria la gestión de los interesados; por lo tanto, la satisfacción de las necesidades negociadas de estos, se estima mediante la siguiente expresión:

Nivel de satisfacción $=$ Percepciones - Expectativas

Al revisar la expresión, es plausible estimar para diferentes momentos, en un proyecto, el nivel de satisfacción, en otras palabras, definir cómo se están gestionando los requerimientos y necesidades de los interesados. Por otra parte, cuando la literatura hace referencia a la gestión exitosa de proyectos, relaciona la habilidad del gerente de proyectos para gestionar y definir los supuestos, restricciones, externalidades y riesgos. Normalmente, los gerentes de proyectos públicos sólo se concentran en el triángulo de hierro, a saber: costos, tiempo y alcance; situación que puede re-direccionar el proyecto hacia otros objetivos. 


\section{Contexto Rastopo, c.}

En términos generales, lo que están expresando los diferentes autores se centra en que la gestión de proyectos debe estar en función de la misión, visión y objetivos estratégicos, por lo tanto, se garantiza que los resultados esperados (objetivos del proyecto) estén alineados con ello, generando valor (bienestar). Es evidente la necesidad de diseño e implementación de oficinas de Gerenciamiento de Proyectos en los entes territoriales, responsabilizándose de la gestión del ciclo de vida del proyecto, del ciclo de vida extendido y del ciclo de vida del gerenciamiento del proyecto. En la Figura 3 se esquematiza el nivel de madurez de este tipo de oficinas.

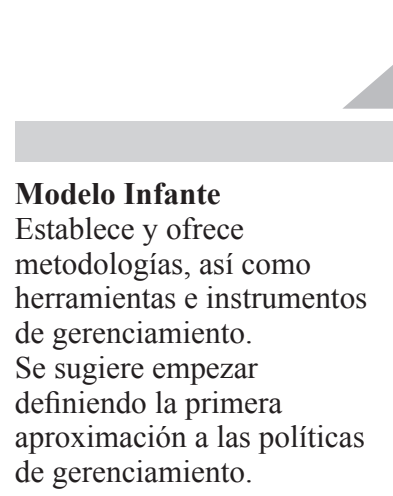

\section{Modelo Adolescente}

Se da inicio a la transferencia de conocimientos, aprendizajes, y experiencias a través de los diferentes proyectos asociados a una o varias unidades de negocio.

Se asegura una adecuada gestión del conocimiento.

\section{Modelo Adulto o Maduro}

Las capacidades y habilidades en concentran en la PMO, la cual provee monitoreo, vigilancia directa a los proyectos, selecciona Gerentes de Proyectos, da asesoría y capacitación.

Figura 3. Madurez de una oficina de Gerenciamiento de Proyectos.

Elaboración propia.

La PMO pública se puede determinar como una unidad de ejecución táctica (Gestión de programas y proyectos, acciones hacia los ejecutores de las iniciativas de proyectos) con acciones de desarrollo de competencias, homologación y asesoría; y unidad de ejecución estratégica (Gestión de portafolios, acciones hacia la alta dirección) con acciones de control y gestión de la estrategia de dirección organizacional de proyectos (OPM) definida para la organización; es decir, portafolio, programas y proyectos. El valor de esta PMO es directamente proporcional al nivel de responsabilidades que gestiona y es asignada por la alta dirección.

La gestión del ciclo de vida de cada proyecto es asunto del gestor encomendado a lograr los objetivos de este y la gestión del ciclo de vida de gerenciamiento es determinado por la PMO en su rol de desarrollo de competencias y asesoría, pero su aplicación es responsabilidad del gestor del proyecto. 
El desarrollo de las habilidades blandas debe ser imperioso en la gestión de proyectos públicos. Aún es, desafortunadamente, muy frecuente encontrar gerentes que han logrado este cargo por el fenómeno conocido como Efecto Halo. En otras palabras, se da por sentado que un buen profesional en un área determinada será un buen gerente en esta misma. Estos gerentes terminan siendo administradores de contratos o simplemente buenos administradores, pero adolecen de una visión estratégica e integradora de todas las variables inmersas en los análisis del entorno y del proyecto; les cuesta imbricar y correlacionar todo lo que sucede al interior y exterior del proyecto. Además, pasan por alto que los proyectos son sistemas abiertos que están en continua interacción con su ambiente, por lo tanto, pueden ser impactados e impactar.

La definición de una política pública de gestión de proyectos permite lograr economías de escala y alcance en el sector público, optimizando la gestión de procesos y operaciones. Este hecho se reflejaría en finanzas más claras, transparencia en los procesos, gestión y reporte de riesgos y mejora en la entrega de productos y/o servicios. Así mismo, la implementación de buenas prácticas gerenciales favorece la moral y satisfacción de los empleados.

\section{Referencias}

Akhmetshina, E. \& Mustafin, A. (2015). Public-private Partnership as a Tool for Development of Innovative Economy. Procedia Economics and Finance, 24, 35-40.

Alborta, G., Stevenson, C. y Triana, S. (2011). Asociaciones público privadas para la prestación de servicios: una visión hacia el futuro. Washington D. C., Estados Unidos: Banco Interamericano de Desarrollo.

Blanco, I. y Gomá, R. (Comp.). (2002). Gobiernos locales y redes participativas: retos e innovaciones. España: Ariel.

Canoy, M., Janssen, M., van Bork, G. y Vollaard, B. (2001). PPS: Een Uidagend Huwelijk, Publiek-Private Samenwerking bij Combinatieprojecten. Holada: Central Planfureau (CPB).

Cavalcante, T., Sousa, M., Milito, C. y Oliveira, P. (2014). Fatores que contribuem para a maturidade em gerenciamento de projetos: o caso de um governo estadual. Revista de administração, 49(2), 415-428. 


\section{Contexto Bestroo, c.}

Crawford, L. \& Helm, J. (2009). Government and governance the value of project management in the public sector. Project Management Journal, 40(1), 73-87.

Evans, M. (2009). Gordon Brown and public management reform -a project in search of a 'big idea'?. Policy Studies, 30(1), 33-51.

Figueroa, G. (2005). La metodología de elaboración de proyectos como una herramienta para el desarrollo cultural. Santiago de Chile: UTEM, FONDART, D.G.I.

Fitsilis, P. \& Chalatsis, T. (2014). Adoption of Project Management Practices in Public Organizations. iBusiness, 6(3), 131-142.

Florescu, M. (2012). Evaluation of the project management organisational ability of public organisations. Annals of Faculty of Economics, 1(2), 197-206.

García Sánchez, I. M. (2007). La nueva gestión pública: evolución y tendencias. Presupuesto y gasto público, 47, 37-64.

Gómez, R. (2012). Gestión de Políticas Públicas: Aspectos Operativos. Revista de la Facultad de Salud Pública, 30(2), 223-236.

Gómez, R., Yepes, C., Rodríguez, F., Roldan, P., Velásquez, W., Lopera, J., .... Agudelo, S. (2012). Manual de gestión de proyectos. Medellín, Colombia: Universidad de Antioquia.

Istrate, L., Marian, L. \& Ferencz, I. (2014). The Use of a Project Managenet Application in Managing Investments in a Public Administration Institution. Prodecia Economics and Finance, 15, 1732-1739.

Jordán, R. y Simioni, D. (Comp.) (2003). Gestión urbana para el desarrollo sostenible de América Latina y el Caribe. Santiago de Chile, Chile: CEPAL, Cooperazione Italiana.

Kapsali, M. (2011). How to implement innovation polices through projects successfully. Technovation, 31(12), 615-626.

Klijn, E. (2009). Public-private partnerships in the Netherlands: policy, projects and lessons. Economic Affairs, 29(1), 26-32. 
Kuprenas, J., Smith, B. \& Madjidi, F. (1999). Implementation Of Project management In Public Engineeringorganization. Journal Of Management In Engineering, 15(3), 69-77.

Lahera, E. (2004). Política y Políticas Públicas. Santiago de Chile, Chile: CEPAL.

Mihaescu, C. \& Tapardel, A. (2013). A Public Administration Based on Project Management. Administration and Public Management, 20, 97-107.

Pierre J. \& Peters, G. (2000). Governance, Politics and the State. London, UK: St. Martin's Press.

Pilkaité, A. \& Chmieliauskas, A. (2015). Changes in Public Sector Management: Establishment of Project Management Offices - A Comparative Case Study of Lithuania and Denmark. Public Policy and Admistration, 14(2), 291-306.

Project Management Institute (PMI). (2017). Guía de los fundamentos para la dirección de proyectos. USA, Atlanta: Project Management Institute.

Pulmanis, E. (2013). Public Sector Project Management Efficiency problems, Case of Latvia. Regional Formation an development Studies, 11(3), 177-188.

Restrepo, C. y Alzate, A. (2013). Aproximación a la Responsabilidad Social Corporativa, con énfasis en el direccionamiento de proyectos. Revista Ciencias Estratégicas, 21(29), 69-85.

Reyes, A. y Gonzalez, L. (2014). Gestión estratégica del talento humano consideraciones de juicio para el desarrollo eficiente de proyectos desde la optica del chao manifiesto. Suma de Negocios, 5(11), 49-61.

Roratto, R. y Dotto, E. (2012). Fatores de risco no gerenciamento de projetos de tecnologia da informação em instituições públicas. Revista de Economia e Administração, 11(3). 386-399.

Schweinheim, G. (1998). Competitividad, agendas locales, gestión municipal y competencias gerenciales públicas en contextos de modernización. En J. C. Venesia. (Ed.), Políticas Públicas y Desarrollo Local (27-42). El Salvador: IDR.

Shiferaw, A. \& Klakegg, O. (2012). Linking policies to projects: the key to identifying the right public investment projects. Project Management Journal, 43(4), 14-26. 


\section{Contexto Pestroos.c.}

Swan, W. \& Khalfan, M. (2007). Mutual objective setting for partnering projects in the public sector. Engineering, Construction and Architectural Management, 14(2), 119-130.

Verweij, S. (2015). Once the shovel hits the ground: Evaluating the management of complex implementation processes of public-private partnership infrastructure projects with qualitative comparative analysis (Tesis $\mathrm{PhD}$ ). The Netherlands: Erasmus University Rotterdam.

Wilenius, M. (2005). Towards the age of corporate responsibility? Emerging challenges for the business World. Futures, 37(2-3), 133-150.

Wu, Y., Huang, Y., Luo, W. \& Li, C. (2014). Construction supervision mechanism for public projects in china: Progress Goal-Oriented perspective American Society of Civil Engineers. Journal of Management in Engineering, 30(2), 205-213.

Yang, C. (2007). Primary influential factors in the management of public transportation projects in Taiwan. Canadian Journal of Civil Engineering, 34(1), 1-11.

\section{Para citar este artículo:}

Restrepo, C. (2020). Aproximación a la compresión del gerenciamiento de proyectos públicos. En-Contexto, 8(12), 47-68. 